\title{
Caracterización de valores que se manifiestan en la interacción docente-alumnos en el programa de administración de empresas de la Universidad de Sucre (Colombia)
}

\author{
Characterization of values that manifest themselves in the teaching-student interaction in \\ the business administration program of the University of Sucre
}

ROJAS, Claudia P. ${ }^{1}$
HERNÁNDEZ, Hugo G. ${ }^{2}$
NIEBLES, William A. ${ }^{3}$

Resumen

La investigación busca caracterizar los valores que se manifiestan en la interacción docente-alumnos, la metodología es descriptiva, no experimental, transversal, de campo. La población estuvo conformada por veinte estudiantes de administración de empresas, se aplicó un cuestionario con 21 ítems. La confiabilidad fue de 0.80 , Los resultados se presentan calculando los puntajes de tendencia central y desviación estándar. Se concluye que existe una interacción coordinada en el aula de clase, visualizando una participación activa para promover los valores.

Palabras clave: valores, caracterización, aprendizaje, integración.

\begin{abstract}
The research seeks to characterize the values that manifest themselves in the teaching-student interaction, the methodology is descriptive, non-experimental, transversal, field. The population consisted of twenty business administration students, a questionnaire was applied with 21 items. Reliability was 0.80 , results are presented by calculating the central trend and standard deviation scores. It is concluded that there is a coordinated interaction in the classroom, visualizing active participation to promote values.

keywords: values, characterization, learning, integration.
\end{abstract}

\section{Introducción}

La globalización y los cambios de la actualidad inciden en el proceso integral de calidad de la formación o educación, razón por la cual, la Organización de las Naciones Unidas para la Educación, la Ciencia y la Cultura (UNESCO) ha enfocado sus proyectos en la búsqueda de componentes que favorezcan la calidad en la educación,

\footnotetext{
${ }^{1}$ Magister en Administración, Especialista en Gerencia del Talento Humano, Docente de la Universidad de Sucre. claudia.rojas@unisucre.edu.co 2

2 Magister en Sistema de Gestión, Especialista en Estudios Pedagógicos, Especialista en Diseño y Evaluación de proyectos, Ingeniero Industrial. Docente Programa de Administración de Empresas Universidad del Atlántico-Colombia. hugoghernandezpalma@gmail.com

${ }^{3}$ Doctor en Ciencias Gerenciales, Magister en Dirección Estratégica, Administrador de Empresas. Docente de la Universidad de Sucre. william.niebles@unisucre.edu.co
} 
pensando en garantizar a la humanidad el manejo del conocimiento y competencias laborales, todo en el marco del desarrollo emocional, el reforzamiento de valores sociales, la eficiencia del desempeño laboral, la solidaridad, colaboración, el patriotismo, entre otros

Ante tal realidad, Parra, Visbal, Duran y Badde (2019), manifiestan que el cambio se da de manera continua y globalizada caracterizado por su capacidad de transformar diferentes aspectos de desarrollo y acción en la vida del hombre, en tal sentido la población es influenciada por modelos de conductas sociales, políticos, económicos, tecnológicos, educativos y culturales, asu vez, estos aspectos también han sido transformados por la capacidad pensante y emotiva del humano, lo que hace que hace se comporte de modo diferente

En este ámbito, se involucran los valores, los cuales juegan un papel primordial en esa forma de comportarse y desenvolverse que tiene el hombre en una sociedad tan cambiante como la actual, la cual conduce muchas veces a la práctica de acciones que están fuera de los parámetros sociales establecidos (Durán, García y Parra, 2018).

Aunado a lo planteado, el proceso de enseñanza - aprendizaje debe promover valores generales para todo los aspectos de la vida del individuo, lo que hace pensar, que sería propicio inducir al estudiante en una formación de valores, lo que permitría el fácil desenvolvimiento del hombre en una sociedad pluralista, con una visión critica y actuando de manera libre, tolerante, honesto, solidario y justo, basados en aspectos concretos de la vida en la sociedad, Rivera et al. (2019) manifiestan que la conducta interpesonal es producto de la interacción, agrupación y el aprendizaje instrumental, soportado por el refuerzo social como elemento trascendental para reforzar y adquirir, precisamente, dicho comportamiento interpersonal.

En este orden de ideas, el proceso de enseñanza está regido por normas legales, procedimientos y reglas correlacionales entre si, como son el caso de la filosofía, psicología y pedagogía, que inciden de tal manera que condicionan e interectuan durante la prendizaje del individuo, generando un trinomio s compuesto por el sistema educativo, el profesor y el estudiante, logrando brindar una formación integral en cualquier persona. En este contexto, Steffens et al. (2017), afirman que para lograr de manera continúa la eficiencia y el mejoramiento en el acto pedagógico es necesario que exista correspondencia entre lo que se exige y se necesita del contexto donde se desarrolla el proceso de enseñanza - aprendizaje.

Steffens, Ojeda, Martínez, Hernández y Moronta, (2018), destacan la importancia de contar con estudiantes de pregrados con pensamiento critico, analíticos y pensantes dentro de un salón de clase, ansiosos por adquirir conocimiento, a través de preguntas e indagaciones, para lograr interiorizar todas las acctivades que se planteen para el aprendizaje y por tanto, para el dominio del conocimiento(García, Durán, Parra y Ramírez, 2018). Desde este punto de vista, se debe considerar la formación de valores como un elemento significativo en el los tiempos modernos, debido a que a nivel global es un tema que causa preocupación por la percepción que tienen en cuanto a la pérdida de valores en las sociedad globalizada, es por esta razón, que se requiere de una preparación y formación en la persona pero de manera conciente sin que tenga incidencia en su normal desarrollo.

Asimismo, Alvarez, Sandoval y Puello (2018), sostienen que la formación es un proceso de liberación a través de planes educativos eficiente en cada nivel, esto indizca que debe sertomado como el elemento eficaz para el aprendizaje del ciudadano que garantice el respeto, la igualdad, tolerancia y solución de conflictos, de acuerdo con las exigencias que demandan la actualidad y los contextos de cada área geográfica donde esté inmerso el individuo.

Además, para Cortina y Conill (2016) continuamente se habla de la progresiva pérdida de valores éticos en la sociedad o en las escuelas y, sin embargo, existen escasas investigaciones en el sector educativo para tratar de profundizar, analizar y solucionar este fenómeno social, que genera grandes y notables fallas en todas las variables asociadas a la calidad educativa de los jóvenes. 
Aunado a lo anterior, en los últimos años, los medios de comunicación social diariamente reflejan hechos de corrupción galopante, cuyos tentáculos alcanzan límites difíciles de imaginar, resultando complejo hablar de valores, tales como: respeto, verdad, honestidad, justicia e igualdad; por la observancia de su debilitamiento de los individuos. En consecuencia, existe la necesidad imperativa del cambio educativo a partir de las transformaciones que enfrenta el hombre del siglo XXI.

Indudablemente que las instituciones educativas, en este caso las universidades, tienen como filosofía la formación integral del estudiante, por lo cual se está propiciando la formación de un ser humano autónomo dotado de principios éticos, para poder entender el qué y para qué de su conducta, en función de las exigencias de la sociedad en la cual vive. No obstante, a pesar de grandes esfuerzos, la constante dinámica de la sociedad actual marcha hacia un exceso de mediciones y mecanizaciones de diferentes manifestaciones en la cotidianidad de la modernidad que provocan distanciamiento de algunas creencias y alteración de valores socioculturales, evidenciados con cierta incertidumbre, en lo que respecta al pensamiento de las personas, de la cultura y la sociedad

Cabe destacar que, en esta orientación social, la moralidad y los principios parecieran estar en discusión (Hernandez, Pitre y Jimenez, 2018); por ello es necesario que el educador reflexione sobre los valores que inculca en los estudiantes, a través de sus clases, los modos de interactuar, relacionarse o el comportamiento que mantiene con ellos, sobre el tipo de aprendizaje que emplea en el aula. En esta perspectiva reflexiva el docente ha de modelar dichos valores e integrarlos en su práctica diaria, mostrándolos y viviéndolos cotidianamente, sin convertirlos en algo excepcional y esporádico.

Por lo tanto, el personal docente debe regir con afecto y amor su trabajo diario, y enseñar a distinguir en el alumnado lo procedente de lo improcedente, lo valioso de lo desechable, de los valores y los antivalores, respectivamente. De esta manera según lo planteado por Moreno, Tezón, Rivera, Duran y Parra (2018), pueda promover su autonomía y desarrollo. Esto lo refuerza Garza (2007), al afirmar que la aplicación de estrategias metodológicas para el desarrollo de valores, por el docente "constituyen herramientas efectivas para el logro de cambios esperados en sintonía con valores como justicia, paz, respeto.

\subsection{Caracterizacion de Valores en el contexto académico.}

La caracterización de los valores para Angelucci et al. (2008), es una dinámica de sintonización que ayuda a conseguir una visión crítica en la vida de los universitarios, influyendo en sus proyectos, sentimientos, y experiencias, con el propósito de conocer cuáles son sus valores. Esta dinámica da a la auto indagación y análisis del propio "yo" no implica doctrinamiento propio y favorece la toma de decisiones, facilitando que el aprendizaje se adquiera y persista duante toda la vida.

Es así como Vargas, Hernandez y Angulo (2018), sostienen que la formación integral de los estudiantes que oriente su conducta al servicio personal o comunitario necesita no solo conociientos si no también competencias profesionales. Así Moreno, Parra, Villasmil, Hernández y Durán (2017), consideran la apropiación y clarificación de conceptos que los oriente a lugar sus metas tales como el emprendimiento son importantes, puesto que, esto le puede dar sentido a su existencia considerados como valores (Durán y Parra, 2014), en tal sentido, en la relación sujero y objeto condicionado por una situación conreta surgen valores representados en las cualidades del individuo, las cuales se hacen evidente en los deseos, anhelos, preferencias o elección de las cosas que mas estima o apego despiertan en las personas, esto hace referencia a los postulados y principios que orientan los comportamientos, actitudes y creencias.

Bajo este contexto, las universidades, al tener una misión aceptada socialmente: educar, deben integrar y conjugar contenidos y valores que sean considerados útiles para el desempeño social del individuo, que a pesar 
de todos los cambios experimentados en las últimas décadas se sigue esperando que las instituciones educativas, en manos de sus docentes enseñen a sus estudiantes y a su vez los estudiantes, aprendan de ellos.

Este precepto tiene como idea, evitar el adoctrinamiento en los estudiantes, mas bien buscar la libertad de pensamiento y elección referente a los valores que cada individuo asuma, de este modo, no se conciben valores superiores, se concibe una jerquización de valores conforme a la configuración que cada individuo haga en función de su propia existencia. Indudablemente, como proceso educativo, la clarificación de valores se fundamenta en tres premisas, lo cual se muestra en el siguiente gráfico 1.

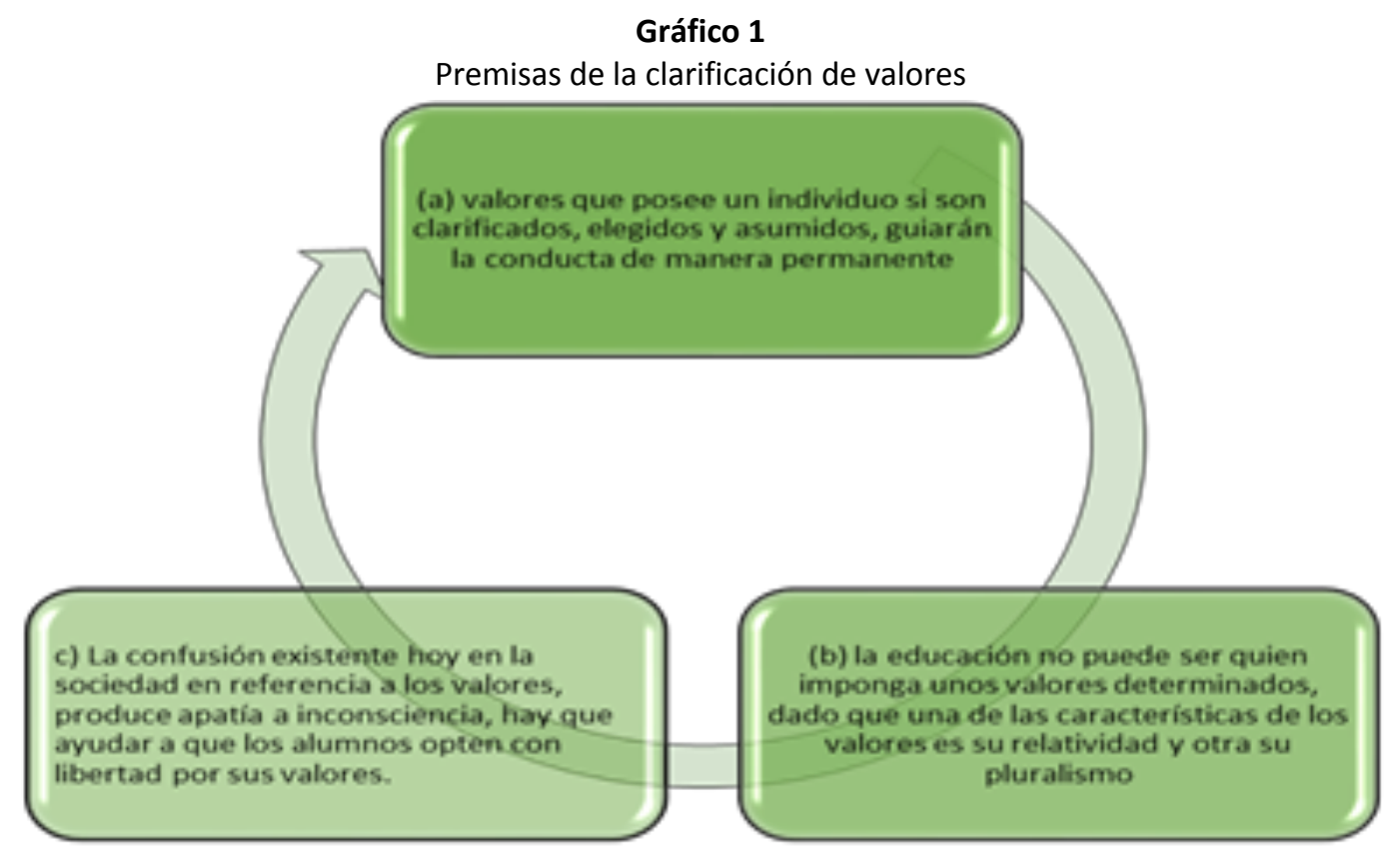

Fuente: Ramos (2001)

Esta actividad es una de las más apropiadas, su autor es Simón (1973), de la Universidad de Massachusetts, y el desarrollo de la misma consta de una serie de estrategias entre las que destacan los siguientes tipos de ejercicios: "Elección entre dos alternativas, votación, jerarquización de alternativas, toma de posición entre extremos, entrevistas y diario, entre otras" (Ramos, 2001, p.77). Estas estrategias se pueden usar en combinación con las áreas académicas como modo de hacer del estudio una actividad totalmente formativa, y no simplemente un ejercicio intelectual.

De esta manera nos encontramos que, según lo expresado por Pitre, Cujia y Hernandez (2018), el proceso de clarificación de valores implica tres momentos fundamentales en la dinámica de interiorización: elección libre de los valores; estimación de los valores elegidos y coherencia en la acción. Estos tres momentos en el proceso de valoración se desglosan en seis pasos o criterios que se consideran imprescindibles para que se pueda comprobar que se ha adquirido o se vive un valor, aspectos que deben ser considerados por el docente al ejecutar tal actividad.

Según Quintana (1998), se ha vuelto cotidiano que existan estudiantes sin un rumbo claro en sus proyecciones de vida, parecieran vivir sin rumbo, sin sentido y con poca motivación,lo cual difculta lograr un análisis sobre la escala de valores que exige esta sociedad. La percepción de vida podría ser diferente si se alcanza una reflexión mediante un método que les permita encontrar en su análisis de vida la clarificación de valores, y comportarse de acuerdo con los mismos. 
Dichos criterios son los siguientes: escoger libremente los valores; seleccionar los valores entre distintas alternativas; analizarlos después de valorar los efectos de cada opción; definir y estimar los valores; comportarse en función con los valores; actuar con estos valores de una manera repetida y constante. Se debe subrayar que los valores son un proceso progresivo personal que abarca toda la vida. Estos pueden ser utilizados como preguntas dentro de un cuestionario para recolectar información, o simplemente como reflexión sobre cómo asumir cada valor.

Las etapas indicadas anteriormente pueden lograr la clarificación de valores, cambian según el momento, dependiendo del profesor, en cuanto a sus destrezas y actitudes, por lo cual debe analizar minuciosamente. Algunos elementos clarificadores para el desarrollo de esta actividad son: se debe crear un clima de libertad, confianza y compromiso para el desarrollo del proceso, a nadie se le debe obligar a participar si no lo desea, se debe respetar la información obtenida y no utilizarla para otras circunstancias diferentes y el docente debe mantener una actitud neutral, es decir, sin parcialización alguna.

Resumiendo, entre las características ideales que deben identificar a un docente para el desarrollo de esta actividad, se pueden considerar las siguientes: autenticidad, compromiso, aceptación, clima apropiado y tener en cuenta el objetivo del educador o su proceso de valoración. Sintetizando, se puede decir que existen infinidad de actividades para desarrollar, que son de gran utilidad práctica y ayudan a ratificar los valores, además de ser medios de socialización y aprendizaje didáctico, pero sin fines evaluativos; la capacidad del docente es el elemento dinamizador capaz de transformar el simple aprendizaje mediante una acción formadora en una buena educación.

Bajo este contexto, la cultura y las experiencias de vida configuran los valores, la concepción del mundo depende de las vivencias individuales, los aspectos sociales o profesionales, el cumulo de información que procese cada persona, entre algunos aspectos, en este contexto Cortina (2007) asumió seis tipos de valores, tales como Libertad, Igualdad, solidaridad, responsabilidad, respeto activo y disponibilidad al diálogo. Según la autora, estos valores pertenecen a la plenitud de lo humano, son libres, ellos depende de cada persona, el encarnarlos o no, pero también son exigibles. En consecuencia, dentro del contexto universitario, es importante educar en valores.

Atendiendo a estas consideraciones, esta investigacion se orientó a caracterizar los Valores que se manifiestan en la interacción docente-alumnos en el programa de administración de empresas de la Universidad de Sucre.

\section{Metodología}

En este estudio, se desarrolló según criterios de la investigación de tipo descriptiva, no experimental, de campo. La población de análisis fue constituida por 20 estudiantes de Administración de empresas de la Universidad de Sucre cursantes del I semestre en segundo periodo del 2019. Para la recolección de la información se utilizó la encuesta, y se diseñó un cuestionario para los estudiantes constituidos por 21 ítems, estructurado con escalas tipo Likert. Los items se muestran a continuación. 
Tabla 1

Relación de items

\begin{tabular}{|c|c|}
\hline \multicolumn{2}{|r|}{ Valores que manifiestan el comportamiento de los estudiantes de administración de empresas. } \\
\hline № & El docente en tu aprendizaje \\
\hline \multicolumn{2}{|c|}{ Indicador: Cooperación } \\
\hline 1 & Asigna trabajos para realizarlos en equipo. \\
\hline 2 & Los estudiantes de mayor conocimiento saben orientar a los otros compañeros \\
\hline 3 & Organiza actividades para ayudar a las personas de la comunidad \\
\hline \multicolumn{2}{|c|}{ Indicador: Respeto } \\
\hline 4 & Orienta a los estudiantes hacia el aprecio por la naturaleza \\
\hline 5 & Toma en cuenta las opiniones de los alumnos \\
\hline 6 & En el grupo da oportunidad a todos. \\
\hline \multicolumn{2}{|c|}{ Indicador: Justicia } \\
\hline 7 & Asigna actividades de acuerdo con el interés de los estudiantes en el tema tratado \\
\hline 8 & Reconoce públicamente a los estudiantes más dedicados al estudio. \\
\hline 9 & Trata a todos los estudiantes sin preferencias. \\
\hline \multicolumn{2}{|c|}{ Indicador: Paz } \\
\hline 10 & Sanciona a los estudiantes por incumplimiento de normas. \\
\hline 11 & Se desarrollan actividades para la integración de equipo \\
\hline 12 & Se enfada cuando los estudiantes no cumplen con sus instrucciones \\
\hline \multicolumn{2}{|c|}{ Indicador: Libertad } \\
\hline 13 & Permite la libre expresión de ideas, en cuanto a los temas tratados en clase. \\
\hline 14 & Toma en cuenta las opiniones de los estudiantes al planificar actividades extra-aulas \\
\hline 15 & Organiza el trabajo en equipo de acuerdo a las necesidades de los estudiantes. \\
\hline \multicolumn{2}{|c|}{ Indicador: Responsabilidad } \\
\hline 16 & Se evalúa el cumplimiento de las actividades en la clase. \\
\hline 17 & $\begin{array}{l}\text { El docente exige a los estudiantes integrarse a las actividades académicas con su } \\
\text { participación activa. }\end{array}$ \\
\hline 18 & Da importancia al cumplimiento de las actividades asignadas. \\
\hline \multicolumn{2}{|c|}{ Indicador: Honestidad } \\
\hline 19 & Genera actitudes de pensamiento libre y firmes. \\
\hline 20 & Prevalece en el aula autenticidad en las relaciones con el docente. \\
\hline 21 & Conversa con los estudiantes sobre los problemas que afectan al ambiente. \\
\hline
\end{tabular}

Fuente: Propia (2020)

La técnica de recolección de datos, fue validado a través del juicio de expertos, y posteriormente se aplicó la prueba piloto, a 10 sujetos con características similares a las de la población, determinando la confiabilidad con el coeficiente de Alfa Cronbach, el cual se aplica para los cuestionarios con escala tipo Likert, obteniendo como resultado rtt: 0,80 .

Luego se procedió a la codificación y tabulación de los instrumentos, presentándose en tablas de tendencias según el baremo de interpretación. Por otro lado, para el tratamiento estadístico se diseñó un baremo (ver cuadro 1) con direccionalidad categórica para la clarificación de valores. El rango se determinó como la diferencia entre la puntuación más alta (PA) menos la puntuación más baja (PB). 
Cuadro 1

Baremo de interpretación

\begin{tabular}{|c|c|}
\hline Rango & Categorías \\
\hline $1,00-1,80$ & Deficiente \\
\hline $1,81-2,60$ & Poco eficiente \\
\hline $2,61-3,40$ & Medianamente eficiente \\
\hline $3,41-4,20$ & Eficiente \\
\hline $4,21-5,00$ & Muy eficiente \\
\hline
\end{tabular}

Fuente: Elaboración propia (2020)

\section{Resultados}

A continuación en la tabla 2, se presentan los resultados referidos al análisis de la estructuración de los valores que se manifiestan en la interacción docente-alumnos en el contexto universitario

Tabla 2

Valores que manifiestan el comportamiento de los estudiantes

\begin{tabular}{|c|c|}
\hline Estadístico & $\begin{array}{c}\text { Valores que manifiestan el } \\
\text { comportamiento del estudiante }\end{array}$ \\
\hline Promedio & 3,8 \\
\hline Moda & 5 \\
\hline Mediana & 4 \\
\hline Desviación estándar & 1,36 \\
\hline
\end{tabular}

Fuente: Elaboración propia (2020)

La tabla 1, permite observar las estadísticas descriptivas para detectar los valores que se manifiestan en la interacción docente-alumnos en universidades, a este respecto en términos generales, la dimensión global presenta una media de respuestas de 3,8 ubicándose en el baremo de interpretación en la categoría eficiente con respecto a la moda ambas se ubicaron en 5 es decir la opción de respuesta más destacada esta se representa con la opción Siempre.

Por otra parte, la mediana se situó en 4, entendida como el dato que divide la distribución en partes iguales, finalmente la desviación estándar fue la medida que tuvo variación asumiendo 1,36, esta información permite referir la dispersión con respecto al promedio, demostrando una baja dispersión de los datos.

Siguiendo con el análisis, la tabla 3 presenta las estadísticas descriptivas para los indicadores de la dimensión, Valores que manifiestan el comportamiento del estudiante, se encontró con respecto al indicador cooperación una media aritmética de 3,5, asumiéndose según el baremo una cooperación eficiente, el valor representado en la moda fue el 5, la mediana se ubicó en 4 y la desviación estándar en 1.2, significando entonces una dispersión muy baja de los puntajes con respecto al promedio.

Tabla 3

Valores que manifiestan el comportamiento de los estudiantes en el aula

\begin{tabular}{|c|c|c|c|c|c|c|c|}
\hline Dimensión & \multicolumn{6}{|c|}{ Valores que manifiestan el comportamiento del estudiante en aula. } \\
\hline Indicador & Cooperación & Respeto & Justicia & Paz & Libertad & Responsabilidad & Honestidad \\
\hline Promedio & 3,5 & 2,9 & 2.4 & 4,6 & 4,7 & 4,5 & 3,8 \\
\hline Moda & 4 & 3 & 2 & 5 & 5 & 5 & 5 \\
\hline Mediana & 4 & 3 & 2 & 5 & 5 & 5 & 5 \\
\hline Desviación estándar & 1,2 & 1,1 & 1,2 & 0,9 & 0,8 & 1,0 & 1,6 \\
\hline
\end{tabular}

Fuente: Elaboración propia (2020) 
Para el indicador Respeto, el promedio se ubicó en 2.9, observándose este valor como de una ación de presencia baja, asimismo la moda y la mediana fueron de 3, la desviación estándar fue de 1,1, significando entonces una dispersión baja de los puntajes con respecto al promedio. Con respecto al indicador Justicia el promedio fue de 2.4, que se interpreta como poco presente, la moda y la mediana fue de 2 , mostrando una tendencia hacia valores negativos, por otro lado, la desviación estándar fue de 1,2, indicando así una dispersión baja de los puntajes en relación al promedio.

Para el indicador Paz, el promedio se ubicó en 4,6 interpretándose este como un valor bastante representativo, pues se encuentra bastante presente, mientras la moda y la mediana se ubicaron en 5 , la desviación estándar fue 0,9 significando una dispersión muy baja de los datos en relación al promedio. Con relación al indicador libertad el promedio fue de 4,7 considerándose muy presente, la moda fue de 5, la mediana fue de 5, la desviación estándar fue de 0,8 significando entonces una dispersión muy baja en relación al promedio.

Asimismo, para el indicador responsabilidad, el promedio se ubicó en 4.5 considerado como muy presente, la moda y la mediana fue de 5, la desviación estándar fue de 1 significando muy baja dispersión de los datos con relación al promedio. Al analizar el indicador honestidad, se observó un promedio de 3.8, este se interpreta como un valor que está bastante presente en el equipo de estudiantes entrevistados, la moda fue de 5, asimismo la mediana asumió el mismo valor, mientras la desviación estándar fue de 1.6 indicando una dispersión baja de los puntajes en correspondencia con el promedio.

Las tendencias asociadas a la caracterizar los valores, se consideró en la mayoría de los procesos de interacción una tendencia positiva, dado que el promedio se ubicó en 3,8 asumiendo, además la mayoría de las posiciones fue hacia una alta presencia en la integración e interacción entre los estudiante y docentes del programa de administración de empresas de la universidad de Sucre. Dado que la moda se ubicó en 5 es decir la opción siempre, fue la más observada por los alumnos, no obstante, la desviación estándar fue la medida que tuvo variación asumiendo 1,36, en virtud de que dos elementos se orientaron hacia líneas de poca presencia en el grupo.

En este sentido, se puede afirmar que los valores se transmiten por contacto, la interacción del docente con el alumno en su convivir son muy importantes, además influye en su internalización, las actitudes y maneras de pensar de los involucrados en este aspecto, despertando el interés para hacer que estos puedan desarrollar el deseo de realización personal, es decir, observando modelos a imitar para cimentar una mejor satisfacción de vida.

Tanto para Esté (2002) como para Durán, Parra y García (2018), los valores constituyen instrumentos que las personas utilizan con el propósito de programar sus acciones y sus maneras de percibir subjetivamente. Asimismo, se evidencia que el sujeto y el grupo construyen sus aprendizajes, conocimientos y proyectos. Pueden tener un desempeño favorable o desfavorable, sin embargo, siempre será vigente con percepción positiva cuando el conocimiento converge entre ellos y negativo, en todo caso, el valor firmemente establecido es necesario para lo que se acepte como verdadero o deseable, lo que les orienta a ser autónomos, tal y como lo ha referido Moreno et al. (2018).

Por lo tanto, los docentes pueden ayudar a que los jóvenes desarrollen los valores y entre estos se menciona que en el trabajo educativo formal e informal, la imaginación creadora juega un papel importante a este respecto. Para Sánchez, García, Steffens y Hernández (2019) y Villarreal, García, Hernández y Steffens (2019), éste educador debe ser capaz de presentar el atractivo del bien, de la honestidad, de la comunicación, de la sencillez de la vida, del compartir, del perdonar; en suma, de todos los valores que han pasado a un segundo plano o prácticamente han desaparecido, por lo que la educación debe ser capaz de generar y de presentar modelos inspiradores. 


\section{Conclusiones}

Luego del análisis se considera una interacción coordinada en el aula de clase, donde estudiantes y docentes tienen participación activa para promover los valores, sin embargo, la prevalencia es la práctica pedagógica formal, asumiendo actitudes en ocasiones discrepantes en cuanto promover la cooperación, el respeto y la justicia.

Atendiendo a las consideraciones anteriores, caracterizar los Valores que se manifiestan en la interacción docente-alumnos en el programa de administración de empresas de la Universidad de Sucre, se observó la prevalencia de unos sobre otros, hay una discrepancia entre los elementos o procesos que se toman en cuenta para promoverlos desde la práctica docente. En este sentido, hay un vacío con respecto a los valores respeto, justicia y honestidad, se ha percibido que no se establecen como parte de la práctica, en virtud de orientar al alumno hacia sus acciones de interacción académica.

Bajo este contexto, existen alguno niveles de deficiencias en aspectos que se manifiestan desde la perspectiva del desarrollo formal de la conducta del alumno, se pudo conocer que existen falencias respecto a las competencias integradoras del docente, las cuales son necesarias para formar ciudadanos en base al respeto, la honestidad, la cooperación y la libertad de pensamientos, por tal motivo se sugiere que deba desarrollarse una serie de actividades y procesos para el desarrollo del docente, y este a su vez pueda promover los valores desde su práctica pedagógica, mejorando la interacción con el estudiante.

\section{Referencias bibliográficas}

Alvarez, A., Sandoval, O., \& Puello, M., (2018), Estrategias pedagógicas para desarrollar competencias ciudadanas en estudiantes de Derecho, Revista espacios, 39(29), 6-16 http://repositorio.cuc.edu.co/bitstream/handle/11323/1613/Estrategias\%20pedag\%C3\%B3gicas\%20para \%20desarrollar\%20competencias\%20ciudadanas\%20en\%20estudiantes\%20de\%20Derecho.pdf?sequence= 1

Angelucci, L., Juárez, J., Dakduk, S., Lezama, J., Moreno, A., \& Serrano, A., (2008), Jerarquía de valores en estudiantes universitarios. Argos, 25(48), 06-20.

Cortina, A., \& Conill, J., (2016), Ethics of vulnerability. In Human Dignity of the Vulnerable in the Age of Rights (pp. 45-61). Springer, Cham

Durán, S., García, J., \& Parra, M., (2018) Diversidad cultural y Fomento de valores en la convivencia ciudadana, Revista Búsqueda, 1(19), Corporación Universitaria del Caribe http://revistas.cecar.edu.co/busqueda/article/view/366

Durán, S., \& Parra, M., (2014), Diversidad Cultural para promover el desarrollo de habilidades sociales en educación superior, Cultura educación y sociedad, 5(1), https://revistascientificas.cuc.edu.co/culturaeducacionysociedad/article/view/995/pdf_212

Esté, A., (2002), Valores y referentes. Caracas, Venezuela. Universidad Central de Venezuela

Garza, E., (2007), Comunicación en Valores. Ediciones Coyoacán.

García, J., Durán, S., Parra, M., \& Ramírez, J., (2018), Gestión del conocimiento: factor estratégico en el desarrollo del talento humano para impulsar la productividad organizacional en pymes, https://www.researchgate.net/profile/Sonia_Duran/publication/333295968_Capitulo_1_Knowledge_man agement_strategic_factor_in_the_human_talent_development_to_promote_organizational_productivity_ 
in_smes/links/5ce5c590a6fdccc9ddc6f64c/Capitulo-1-Knowledge-management-strategic-factor-in-thehuman-talent-development-to-promote-organizational-productivity-in-smes.pdf

Hernandez, H., Pitre, R., \& Jimenez, M. (2018). Continuous Improvement for Colombian Universities through Innovation in Information Management. Contemporary Engineering Sciences, Vol. 11, 2018, no. 83, 4095 4103

Moreno, Z., Parra, M., Villasmil, M., Hernández, B., \& Durán, S., (2017), Importancia del Pensamiento Estratégico y Acciones Estratégicas para impulsar el emprendimiento social en las universidades venezolanas. Revista Espacios, 38(45), https://www.revistaespacios.com/a17v38n45/a17v38n45p04.pdf

Moreno, M., Tezón, M., Rivera, T., Duran, S., \& Parra, M., (2018), Autoestima: Desarrollo de la autonomía personal en estudiantes del área técnica, Revista Espacios, 39 (46), https://www.revistaespacios.com/a18v39n46/a18v39n46p06.pdf

Parra, M., Visbal, O., Duran, S., Badde, G., (2019) Calidad de la comunicación y actitud de los empleados ante procesos de cambio organizacional Interdisciplinaria, Centro Interamericano de Investigaciones Psicológicas y Ciencias Afines, 36(1), 155-170, doi: https://doi.org/10.16888/interd.2019.36.1.11

Pitre, F., Cujia, S., \& Hernandez, H., (2018) Challenges of Education in the Knowledge Society: Components for Universal Education. Contemporary Engineering Sciences, Vol. 11, 2018, no. 82, 4085 - 4093

Quintana, J., (1998), Pedagogía axiológica. Madrid: Dykinson.

Ramos, M., (2001), Para Educar Valores, Teoría y Práctica. Venezuela. Paulino.

Rivera, J., Lay, N., Moreno, M., Pérez, A., Rocha, G., Parra, M., Durán, S., \& Redondo, O., (2019), Programa de entrenamiento para desarrollar habilidades sociales en estudiantes universitarios. Revista espacios. 40 (31), 10, https://www.revistaespacios.com/a19v40n31/a19v40n31p10.pdf

Sánchez, M., García, J., Steffens, E., \& Hernández, H., (2019), Estrategias Pedagógicas en Procesos de Enseñanza y Aprendizaje en la Educación Superior incluyendo Tecnologías de la Información y las Comunicaciones. Información tecnológica, 30(3), 277-286, doi: https://dx.doi.org/10.4067/S0718-07642019000300277

Simón, S., (1973), Los valores y la enseñanza. Religious Education, 68 (2), 183-194.

Steffens, E., Ojeda, D., Martínez, J., Hernández, H., \& Moronta, Y., (2018), Presencia del pensamiento crítico en estudiantes de educación superior de la Costa Caribe Colombiana, Revista Espacios, 39(30), 1, https://www.revistaespacios.com/a18v39n30/a18v39n30p01.pdf

Steffens, E., Ojeda, D., Martínez, O., García, J., Hernández, H., \& Marín, F., Niveles de pensamiento crítico en estudiantes de Universidades en Barranquilla (Colombia) (2017), Revista Espacios, 38 (30), 5 Recuperado de http//www.revistaespacios.com/a17v38n30/a17v38n30p05.pdf

Unesco. Foro mundial de educacion. (2015). Declaración de Incheon. Educación 2030: Hacia una educación inclusiva y equitativa de calidad y un aprendizaje a lo largo de la vida para todos. ED/WEF2015/MD/3. Extraído de http://unesdoc. unesco. org/images/0023/002331/233137s. pdf.

Vargas, C., Hernandez. H., \& Angulo, F., (2018). Information Management as a Quality-Building Element in Higher Education Institutions. Contemporary Engineering Sciences, Vol. 11, 2018, no. 87, 4311 - 4319 
Villarreal, S., García, J., Hernández, H., \& Steffens, E., (2019), Competencias Docentes y Transformaciones en la Educación en la Era Digital, Formación universitaria, 12(6), 3-14, doi: https://dx.doi.org/10.4067/S071850062019000600003

Esta obra está bajo una Licencia Creative Commons

Attribución-NoCommercial 4.0 International

\section{(cc) BY-NC}

